\title{
Factores de riesgo cardiovascular y síndrome metabólico en población laboral (sector industrial). Estudio de cohortes a 5 años.
}

\author{
Cardiovascular risk factors and metabolic syndrome \\ working population (industrail sector). Cohort a 5 years.
}

\section{Carlos Alberto Názara}

PhD, Internista y médico de familia C.S. Marín Eoxi Pontevedra/Salnés.

Universidad de Vigo. Facultad de Biología. Departamento de Bioquimica, Genética e Inmunología.

El Síndrome Metabólico (SM), es una entidad clínica de agrupación de factores de riesgo cardiovascular (CV) en el que subyace un trastorno del metabolismo hidrocarbonado y que supondría un mayor riesgo de enfermedad CV que el debido a la suma de todos los factores que lo constituyen, de modo independiente.

A día de hoy no se acepta que sea un diagnóstico a consignar en la historia clínica del paciente, si bien se trataría de una herramienta en el abordaje del riesgo CV del individuo.

Hay varios criterios, basados en consenso, para el diagnóstico de SM. Aquellos que resultan válidos y se toman como referencia son: OMS, EGIR, NCEP-ATPIII,IDF.

La prevalencia del SM es muy variable, oscilando en Europa entre un 7\%-36\%, dependiendo de la edad, sexo, zona geográfica, grupo étnico y enfermedades subyacentes. Dicha variabilidad aplica, como cabe suponer, a nuestro país.

El SM es un auténtico problema sanitario, debido a la morbilidad y mortalidad de las poblaciones que lo presentan, siendo lo más destacado, su asociación con el desarrollo de Diabetes Mellitus tipo 2 (DM2). Desde el punto de vista clínico-asistencial y de salud pública, interesa su identificación temprana para una actuación de prevención de la enfermedad CV, a partir del supuesto riesgo que entraña, más allá de la mera alteración del metabolismo hidrocarbonado, y debido al riesgo relativo añadido de cada uno de sus componentes.

Los objetivos principales del estudio son: Realizar una descripción, en función de variables antropométricas, de una muestra de población laboral; analizar las variables relacionadas con el SM y su distribución y prevalencia en dicha muestra; estimar la prevalencia del SM en población laboral española, basándose en un estudio muestral de población laboral del sector industrial; efectuar una aproximación al estudio del Riesgo Cardiovascular global en la población considerada desde el contexto de un trastorno metabólico subyacente.

Los objetivos secundarios a conseguir son: Analizar otras variables de implicación en el SM (obesidad y colesterol); estimar la prevalencia de hipercolesterolemia en la pobla- ción de estudio; analizar la variabilidad de los factores de riesgo considerados en el período de observación; efectuar una aproximación a la morbimortalidad cardiovascular del colectivo, durante el período de observación.

El trabajo se realizó en el marco de un esquema de reconocimiento periódico anual de salud laboral, que reunió una muestra de 221 personas, que fueron seguidas a lo largo de 5 años. La cohorte vino definida por la aceptación para participar en el estudio de salud laboral y el consentimiento tácito para el uso (de forma anónima) de los datos relativos a dicho examen de salud.

Se consideraron las siguientes variables del estudio: Edad, peso, talla, IMC, circunferencia abdominal, presión arterial, glucosa plasmática basal, colesterol total, colesterol HDL, triglicéridos.

Se aplicaron la prueba de Kolmogorov-Smirnov (distribución normal) y la de Levene (homogeneidad de varianzas), seguido de pruebas paramétricas o no paramétricas según correspondiera (prueba $\mathrm{T}$ de student o la $\mathrm{U}$ de Mann-Whitney y la prueba ANOVA de 1 factor). También la prueba de Chi cuadrado y el estadístico exacto de Fisher para la comparación de variables categóricas. En todos los casos se calculó el intervalo de confianza del 95\%. Todos los análisis estadísticos se realizaron empleando el paquete estadístico SPSS 17.0 para Windows.

La muestra constaba de 221 personas de ambos sexos. $86 \%$ eran varones y el $14 \%$ mujeres. La media de edad fue de 38.4 años, con un rango de 19-58 años. A lo largo de los 5 años hubo una variabilidad mínima en cuanto a la distribución por sexo y edad. La distribución no seguía un patrón normal.

Presentaban sobrepeso/obesidad el 62,9\% de los individuos y de entre ellos, el $22.1 \%$ presentaban obesidad de grado II-III.

La prevalencia del SM, para ambos sexos (no se estableció la diferencia por sexos por el bajo porcentaje de mujeres en la muestra), fue del $23,1 \%$ por criterios ATPIII y del 29,4\% por criterios IDF (mayor porcentaje por criterios más restrictivos en la circunferencia abdominal) 

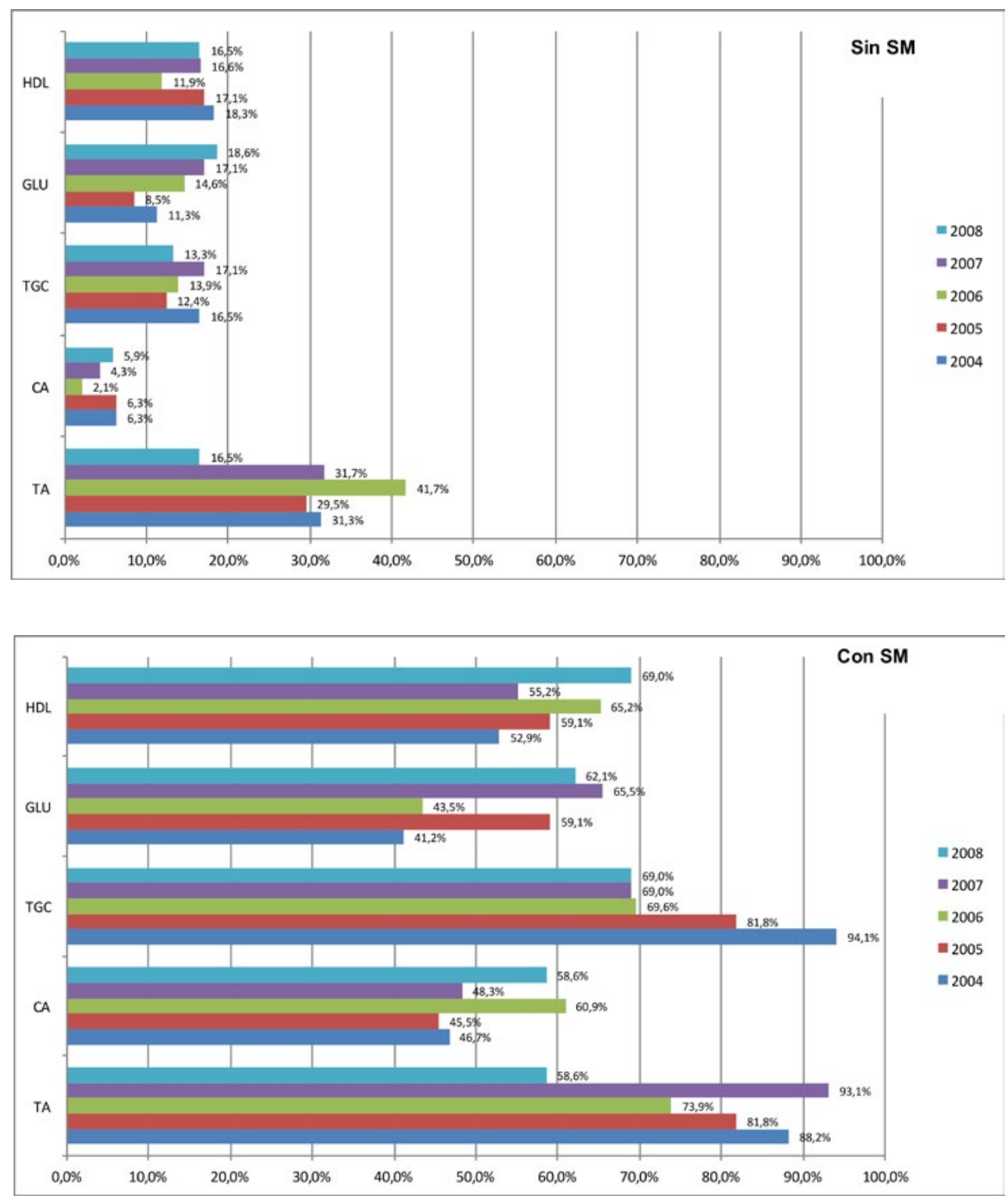

Según Criterios ATPIII, el gran componente del SM (en cuanto a su participación porcentual), a excepción del año 2008 es la presión arterial $(\mathrm{PA})$ elevada y cobran una gran importancia ponderada las cifras bajas de colesterol HDL y los valores altos de triglicéridos.

Cabe resaltar la importante diferencia existente en cuanto a la alteración de la circunferencia abdominal (CA) entre los individuos que tienen SM con respecto a los que no tienen
SM, indicando así la gran participación que tiene como indicador de SM la CA.

La PA, que es la variable más comúnmente alterada, no presenta una marcada asociación con SM (demostrado por los estudios de contingencia para la estimación del riesgo de cada variable), siendo así la CA y los triglicéridos los que más determinan la presencia de SM. 